\title{
Estudio de las creencias de una futura maestra sobre la construcción del repertorio lingüístico a través de narrativas multimodales
}

\author{
Míriam Cabré Rocafort \\ Universitat de Barcelona, España
}

Artículo recibido 5 October 2015, aceptado 18 March 2016, versión final 21 March 2016

DOI: http://dx.doi.org/10.5565/rev/jt13.652

\begin{abstract}
Resumen
La narrativa es una práctica reflexiva que permite (re)pensar y atribuir sentido a las experiencias y comprender la complejidad de los procesos de enseñanza y aprendizaje de lenguas. El objetivo es analizar las creencias de una maestra en formación inicial sobre la construcción del repertorio lingüístico e indagar sobre su proyección en las futuras prácticas docentes. La articulación de las creencias se lleva a cabo a partir de narrativas multimodales, de las que se explora y verifica su eficacia como herramienta metodológica.

El estudio muestra que hay creencias arraigadas y otras más susceptibles de desestabilizar. El sujeto ha construido nuevos marcos interpretativos sobre la educación en lenguas: el foco que había sobre el dominio de una lengua se ha desplazado hacia la noción de desarrollo del repertorio lingüístico. Los procesos reflexivos han posibilitado atribuir sentido a sus experiencias y movilizar o desestabilizar creencias que guiarán sus futuras actuaciones en el aula.
\end{abstract}

Palabras clave: práctica reflexiva, creencias, narrativas multimodales, repertorio lingüístico, formación inicial de maestros, enseñanza y aprendizaje de lenguas

\section{Resum}

La narrativa és una pràctica reflexiva que permet (re)pensar $\mathrm{i}$ atribuir sentit a les experiències i comprendre la complexitat dels processos d'ensenyament i aprenentatge de llengües. L'objectiu és analitzar les creences d'una mestra en formació inicial sobre la construcció del repertori lingüístic i indagar sobre la seva projecció en les futures pràctiques docents. L'articulació de les creences es fa a partir de narratives multimodals, de les quals s'explora i verifica la seva eficàcia com a eina metodològica.

L'estudi mostra que hi ha creences molt arrelades i d'altres més susceptibles de desestabilitzar. El subjecte ha construit nous marcs interpretatius sobre l'educació en llengües; així, el focus que hi havia sobre el domini d'una llengua s'ha desplaçat cap a la noció de desenvolupament del repertori lingüístic. Els processos reflexius han fet possible atribuir sentit a les seves experiències i mobilitzar $i$, en alguns casos, desestabilitzar creences que guiaran les seves futures actuacions a l'aula.

Paraules clau: pràctica reflexiva, creences, narratives multimodals, repertori lingüístic, formació inicial de mestres, ensenyament i aprenentatge de llengües 


\begin{abstract}
Narrative is a reflective practice that allows people to (re)think and to give a sense to their experiences and to understand the complexity of language teaching and learning processes. The aim is to analyse beliefs of a teacher in training about the construction of the linguistic repertoire and to look into its projection in their future teaching. The data collection is carried out through multimodal narratives, whose efficacy has been explored and confirmed as a methodological tool.

The study proves that there are rooted beliefs and other beliefs that are more susceptible to be destabilized. The subject has built new interpretative frameworks about language education. The focus on proficiency in a language has been displaced by the notion of linguistic repertoire development. The reflective processes have permitted to give a sense to her experiences and to mobilize or destabilize some beliefs that will guide her future actions and behaviour in classroom.
\end{abstract}

Key words: reflective practice, beliefs, multimodal narratives, linguistic repertoire, initial teacher training, teaching and learning languages

\title{
Introducción
}

El marco teórico se divide en tres apartados: la investigación biográfica-narrativa, la esfera multimodal y el concepto de creencias. Éstos se relacionan, respectivamente, con el enfoque del estudio, la metodología y el concepto objeto de análisis.

\section{La investigación biográfica-narrativa en educación}

Según Toolan (2001), la narrativa es el proceso de relatar experiencias distantes en términos de espacio y tiempo a través de un narrador a una audiencia, por lo que es una actividad social (narrador-audiencia) y cognitiva (atribución de sentido a las experiencias). El individuo se construye en su entorno a través del lenguaje y la consideración de marcos interpretativos socialmente construidos. Las personas necesitan narrar y narrarse, para adquirir consciencia del significado de sus acciones y decisiones. Esto significa que su situación no es estática, sino sujeta a una historia que adquiere sentido cuando es narrada. Narrar es interpretar, ya que conlleva autoconsciencia respecto la propia experiencia y siempre hay una perspectiva desde la que se 
narra. Las narraciones de la realidad no son completas, porque siempre están abiertas a nuevas interpretaciones (Palou y Fons, 2011).

Este estudio se enmarca dentro de la investigación biográfico-narrativa que es una perspectiva de la investigación educacional. Las narrativas son 'espacios de reinterpretación de las propias experiencias en vista de nuevos marcos de referencia' (Bolívar, Domingo y Fernández, 2001, p. 18). Para llevar a cabo este tipo de investigación es necesario hacer una doble descripción: retratar la realidad interna del sujeto (microcosmos) e inscribirla en un contexto externo (macrocosmos).

Desde la perspectiva etnosociológica, Bertaux (2005) diferencia entre 'historia de vida' (life history), que es la historia vivida por una persona, y 'relato de vida' (life story), que es el relato de toda o una parte de esta historia que se hace a petición de un investigador con una finalidad concreta. Pensar el individuo en función de la identidad narrativa otorga importancia a las historias personales en su construcción identitaria, así como también en la proyección de su identidad en la esfera social (Ricoeur, 1995).

En este estudio, se utiliza la narración de carácter introspectivo (Martuccelli, 2013), que se centra en el análisis de las experiencias y ofrece una visión interior vinculada a la relación del sujeto con las lenguas. El plurilingüismo es un rasgo de su trayectoria lingüística, ya que se utiliza para describir el repertorio de lenguas y competencias lingüísticas de un individuo (Prasad, 2014). Los relatos permiten expresar a un receptor la propia experiencia, es decir, poner el 'yo' en escena con un propósito. Cuando este 'yo' incide en lo que hace y piensa se favorece la metacognición (Palou y Fons, 2011).

\section{La esfera multimodal y las narrativas visuales}

El uso de métodos biográfico-narrativos tiene una larga tradición, especialmente los orales y escritos (biografías, relatos de vida y entrevistas). Prasad (2014) apunta la necesidad de estudiar las creencias a través de métodos narrativos visuales. En ellos, las voces de los sujetos están presentes y es posible analizar cómo se desarrollan los procesos de atribución de significado. En este estudio, el acceso a las creencias no sólo se lleva a cabo mediante narrativas escritas -relato 
de vida lingüística-, sino también a través de la narrativa visual -autorretrato lingüístico-, por lo tanto, se trabaja con métodos multimodales.

La noción de narrativas se ha ampliado en términos de modos y significados: puede ser escrita, oral o visual; o bien multimodal, es decir, la combinación de texto e imagen (Kalaja, Dufva y Alanen, 2013). Estas producciones tienen la ventaja de explicitar hechos que no pueden ser narrados con palabras. La naturaleza multimodal de los datos hace que se obtengan formas de expresión, experiencia y atribución de sentido diversas.

Las relaciones entre las narrativas visuales y la esfera social son evidentes, ya que plasman representaciones sociales culturalmente construidas, que se han adquirido en el ámbito familiar, escolar o profesional (Perregaux, 2011). En las narrativas visuales la atención está focalizada en el modo de relacionarse de los diferentes componentes, para ofrecer una instantánea de una situación o el panorama de un momento particular (Kalaja, Dufva y Alanen, 2013) ligado a la relación del individuo con las lenguas.

\section{El concepto de 'creencias' en la investigación educativa}

La enseñanza entendida como actividad de pensamiento ha impulsado la investigación sobre creencias en el ámbito educativo. De estos estudios destaca la falta de consenso sobre el concepto 'creencias' (Borg, 2003 y Woods, 2006), que conlleva confusión terminológica (teorías, concepciones, pensamientos, creencias, representaciones o saberes).

Woods (2006) habla de los BAK 'as an integrated network of beliefs, assumptions and knowledge', es decir, interrelaciona creencias, presuposiciones y conocimientos. Explica que son conceptos asociados, que implican el desarrollo de estructuras dinámicas y comprensivas de los fenómenos en sus contextos. Su distinción sólo es posible en un contexto particular, a través del planteamiento de una pregunta.

Las creencias se encuentran en constante proceso de re-negociación y son el resultado de procesos de interacción. Los discursos de las personas están ligados a los discursos de la sociedad, por lo que las creencias son interdiscursivas y los discursos polifónicos (Bakhtin, 1982). Éstas tienen las siguientes características (Gabillon, 2012): están contextualizadas; tienen una dimensión personal y otra social; se mantienen explícitas o implícitas; son un marco de 
referencia para la tarea docente; aunque son resistentes al cambio, pueden acontecer dinámicas con la modificación de los contextos; y son complejas, sistemáticas y eclécticas. Los factores que inciden en su desarrollo de mayor a menor impacto son: la propia experiencia como aprendiz; la formación inicial de maestros; las experiencias en docencia; la interacción social; y las lecturas. Las creencias vinculadas al pasado como aprendiz tienen una mayor incidencia en la actuación docente que la información teórica aprendida durante la formación superior, la cual es utilizada para confirmar sus creencias.

En los procesos de decisión no todas las creencias tienen la misma importancia. Existen unas creencias base (core beliefs), que son influyentes, dominantes, resistentes, sociales, explícitas y sistemáticas; y unas creencias periféricas (peripheral beliefs), que están subordinadas a las creencias base y que son diversas, variables, personales, implícitas y complejas (Gabillon, 2012, p. 195-199). La movilización de las creencias se consigue mediante la interacción social en nuevos contextos, ya que las creencias son situadas, dinámicas y multivoces (Alanen, 2006). Cualquier dato de las narrativas ofrece información importante, pero nunca son la verdad completa porque no son conceptualmente posibles. En este estudio se aportan ideas significativas sobre las creencias de una futura maestra y se deja la puerta abierta a otras interpretaciones fruto de una narración que siempre continúa.

\section{Objetivos y preguntas de investigación}

El objetivo general es:

Analizar las creencias de una maestra en formación inicial sobre la construcción del repertorio lingüístico, a partir de la articulación de sus experiencias mediante narrativas multimodales.

Los objetivos específicos son:

- Examinar las relaciones entre las creencias del relato de vida lingüística y de la narrativa visual sobre la relación del sujeto con las lenguas.

- Explorar la eficacia de las narrativas multimodales como recurso metodológico para movilizar creencias sobre la formación del repertorio lingüístico.

- Identificar la proyección de las creencias de la futura maestra alrededor de la enseñanza y el aprendizaje de lenguas en sus futuras prácticas docentes.

Las preguntas de investigación son:

Bellaterra Journal of Teaching \& Learning Language \& Literature. 9.3 (Aug-Sept 2016)

ISSN 2013-6196 
- ¿Qué creencias articula, a través de procesos de reflexión, una maestra en formación inicial sobre la construcción del repertorio lingüístico?

- ¿En qué medida las narrativas multimodales son un recurso metodológico eficaz para movilizar las creencias de la futura maestra?

- ¿Cómo el sujeto proyecta sus creencias alrededor de la enseñanza y aprendizaje de lenguas en sus futuras prácticas docentes?

\section{Metodología}

\section{Instrumentos y procedimiento}

El paradigma de investigación en el que se enmarca esta investigación es el cualitativointerpretativo, ya que pertenece a la investigación biográfica-narrativa en educación. Se trata de un estudio de caso, del cual se muestra el corpus de datos completo. Se utilizan dos métodos de recogida de datos: el relato de vida lingüística y la narrativa visual (figura 1).

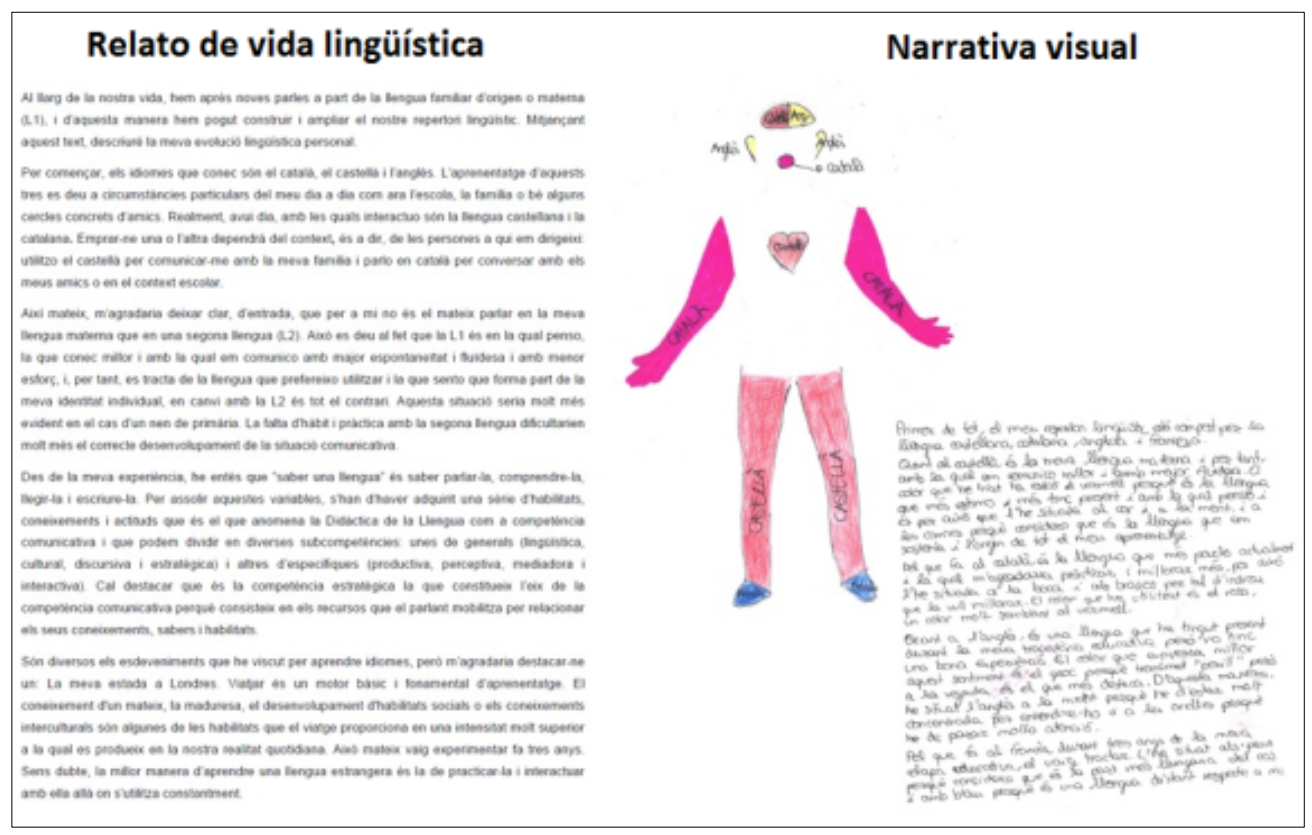

Fig. 1. Corpus de datos

Desde el campo de la sociología, Martuccelli (2013) introdujo el concepto de narración introspectiva, el cual es utilizado en este estudio para relatar la relación del individuo con las lenguas. El relato de vida lingüística es un texto centrado en la construcción del repertorio lingüístico en el pasado, presente y futuro. Se habla de relato en tanto que ha sido solicitado por una investigadora y porque permite entrar en diálogo con lo que para el sujeto tiene significado. 
Además, se explicita su tipo, 'de vida lingüística', ya que el énfasis recae en cómo la persona se sitúa respecto las lenguas. En la narración se incluyen temas como las lenguas que conoce y sus formas de aprendizaje, entre otros.

Para la narrativa visual se pidió ilustrar dentro de una silueta humana las lenguas con las que se interactúa e interpretar el dibujo por escrito. La representación visual muestra cómo se siente la persona en relación con las lenguas en el presente. Durante la construcción de las narrativas se procedió de manera que se dio a la futura maestra un conjunto de consignas sobre el tema para orientarla en el proceso de escritura y representación, pero se le dio libertad para escoger sobre qué y cómo quería escribir y dibujar. No obstante, los datos que se han obtenido surgen del diálogo entre ésta y la investigadora, que precedió la elaboración de las narrativas, por lo que su voz está presente en el proceso de interpretación.

\section{Participantes}

Este trabajo es un estudio de caso, que se enmarca dentro de una investigación más amplia en la cual participaron quince maestros en formación inicial. Las características principales del sujeto son: estudiante del Grado en Educación Primaria (Universitat de Barcelona); de género femenino; y de 18 años. Es exalumna de la investigadora, lo que facilitó el contacto para participar de forma voluntaria en la investigación. Su elección ha estado motivada por la calidad de sus narrativas, en relación con los objetivos del estudio.

\section{Análisis de datos}

Los criterios que se han utilizado para el análisis del relato de vida lingüística se centran en tres dimensiones del texto (Palou y Fons, 2011): la interlocutiva analiza el contexto en el que se enmarca la narración; la temática tiene como objetos de estudio los temas, la relación del individuo con las lenguas y la influencia de su estatus; y la enunciativa examina el posicionamiento enunciativo del sujeto respecto a lo narrado. También se focaliza la atención en las creencias y su tipo: base o periférica.

Los criterios de análisis de la narrativa visual tienen en cuenta los siguientes aspectos: la organización de los datos (Kalaja, Dufva y Alanen, 2013); los elementos representados (Melo- 
Pfeifer y Schmidt, 2012); la dimensión contextual (Pavlenko, 2007); el sistema de creencias (Gabillon, 2012), del que se analiza su tipo (base o periférica) y las contradicciones o corroboraciones que presentan en contraste con las del relato; otras informaciones composicionales, como el color; y la relación entre la interpretación escrita de la futura maestra sobre la narrativa visual y la representación objeto de estudio.

El análisis exhaustivo de las dimensiones del relato y la narrativa visual no se plasma en el siguiente apartado, sino que se focaliza la atención en las creencias movilizadas que están ilustradas con fragmentos del texto y la representación. A continuación, en la figura 2 se puede observar un ejemplo de análisis del bloque temático 1 del relato; y en la figura 3 una muestra del análisis de la narrativa visual.

TEMA 1. Contextualització $\mathbf{i}$ introducció a la temàtica del relat: la pròpia vida lingüistica

Al llarg de la nostra vida, hem après noves parles a part de la llengua familiar d'origen o materna (L1), i d'aquesta manera hem pogut construir i ampliar el nostre repertori lingoístic. Mitjançant aquest text, descriuré la meva evolució linguistica personal.

\begin{tabular}{|c|c|c|}
\hline DIMENSIO INTERLOCUTIVA & DIMENSIÓ TEMÁTICA & DIMENSIO ENUNCIATIVA \\
\hline 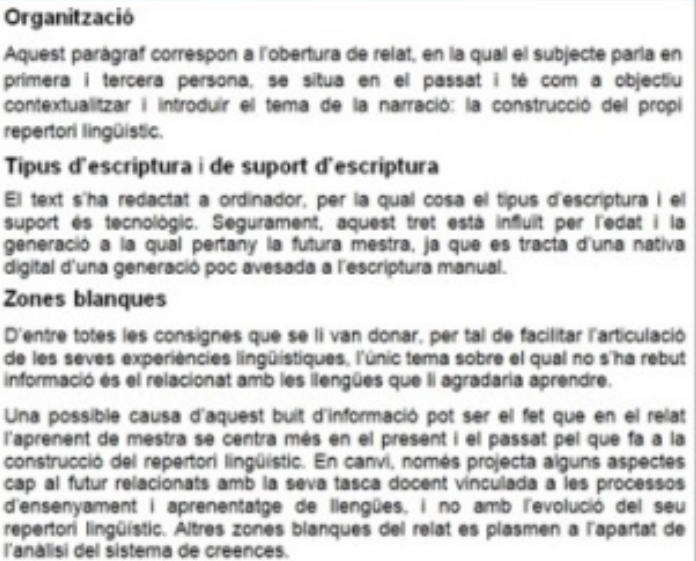 & $\begin{array}{l}\text { Temàtiques } \\
\text { Aquesta is la primera sequencia } \\
\text { tematica de les deu que to el relat } \\
\text { 1. Contextualizacio I introduccio } \\
\text { a. ia tomatica dal rolat la propia } \\
\text { vida linguistica. }\end{array}$ & 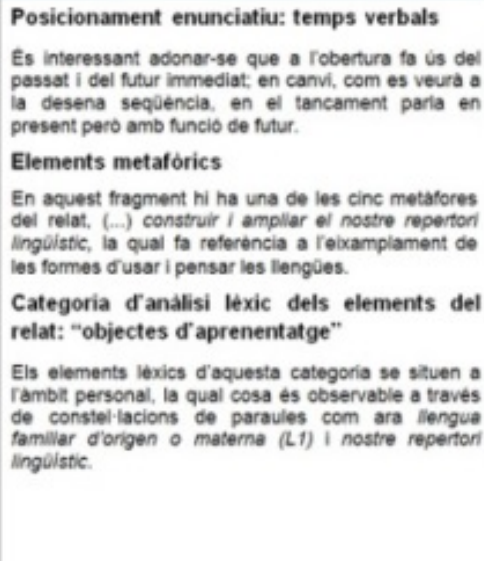 \\
\hline
\end{tabular}

Fig. 2. Ejemplo de análisis de un bloque temático del relato 


\section{Distribució dels elements del dibuix: les parts del cos i les llengües}

La distribució de les llengūes a les diferents parts del cos també és un altre indicador de les formes de relació de la futura mestra amb les llengües. El castellà apareix tres vegades, una a cadascuna de les tres principals zones del cos: cap, tronc i extremitats, perquè és la seva llengua materna i de referència.

A la zona cerebral, concretament està situada a l'hemisferi dret, que està especialitzat en la informació visual i espacial i que processa les dades

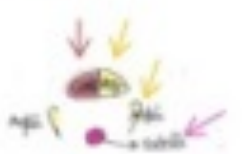
simultàniament. Això està relacionat amb el fet que aquesta és la seva llengua materna, la qual està molt present al seu entorn familiar $\mathrm{i}$ a la societat en què està immersa. A més, és possible que I'hagi conegut a través de formes d'aprenentatge més aviat visuals $\mathrm{i}$ experimentals en els diferents contextos socials. A la part del trone està col-locada al cor pel valor afectiu que li atribueix. A les extremitats inferiors esta situada a les cames, pel fet de ser una llengua que sempre l'acompanya i que l'ajuda a avançar diàriament.

El català apareix a dues parts del cos: la boca i les extremitats superiors. La seva col-locació respon a la voluntat de la futura mestra d'expressar que aquesta és una llengua d'us habitual (situada a la boca), la qual li ofereix recursos per relacionar-se en situacions comunicatives (situada a les extremitats superiors).

Pel que fa a l'anglès, també apareix dues vegades, però a llocs diferents. El trobem a l'hemisferi esquerra, el qual està especialitzat en el llenguatge $\mathrm{i}$ el qual processa la informació de forma seqüencial. Això es relaciona amb el fet que aquesta és una de les seves segones llengües, la qual ha après, majoritàriament, en contexts formals i reglats. L'anglès també està situat a les orelles, la qual cosa apel-la al grau de destresa en comprensió oral o a l'agudesa auditiva que necessita desenvolupar per a comprendre aquesta segona llengua.

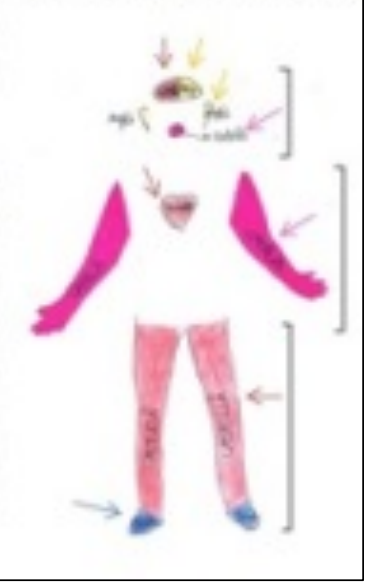

Fig. 3. Ejemplo de análisis de un aspecto de la narrativa visual 


\title{
Análisis y resultados de las creencias de la futura maestra
}

\author{
Del relato de vida lingüistica
}

\section{SISTEMA DE CREENCIAS \\ En el relato hay 6 creencias base $(C B)$ que tienen asociadas creencias periféricas (CP):}

\begin{abstract}
CB 1: Aprender una lengua es aprender a interactuar dentro del ámbito de la comunicación.
A lo largo de nuestra vida, hemos aprendido nuevas hablas, a parte de la lengua familiar de origen o materna (L1), $y$ de esta manera hemos podido construir y ampliar nuestro repertorio lingüístico.

CP 1.1: Entre otras cosas, saber una lengua es saber hablarla y comprenderla.

A partir de mi experiencia, he entendido que 'saber una lengua' es saber hablarla, comprenderla, leerla y escribirla.

CP 1.2: La mejor manera de aprender una lengua extranjera es hablar e interactuar allí donde se utiliza.

Sin duda, la mejor manera de aprender una lengua extranjera es la de practicarla e interactuar con ella alli donde se utiliza constantemente.

CP 1.3: Las actividades de lengua en la escuela tienen que ser pragmáticas y dinámicas, con la intención que el alumnado pueda afrontar situaciones comunicativas reales.

Las actividades de lengua que se tendrían que proponer en la escuela tendrían que ser dinámicas y pragmáticas, con la intención que el niño pueda afrontar situaciones comunicativas actuales reales.
\end{abstract}

CP 1.4: La adopción del uso social de la L2 depende de la presencia de hablantes.

(...) el aprendizaje de 'la otra' lengua es una experiencia compartida, ya que la adopción del uso social de 'la otra' lengua depende de un seguido de condicionantes, sobre todo de la presencia de hablantes.

CP 1.5: La misión de la maestra es crear y desarrollar la competencia estratégica del alumnado, que se entiende como la capacidad de afrontar situaciones comunicativas auténticas.

El objetivo de esta asignatura es la creación y desarrollo de una competencia estratégica rica y diversa en los alumnos, y capaz de hacer crecer el resto de competencias.

En las CP 1.3 y 1.5 la futura maestra se proyecta hacia las futuras prácticas docentes, ya que se centra en el papel y la función de la maestra y en las actividades de lengua.

CB 2: La maestra de Educación Primaria tiene que ser un hablante ideal y una buena referente para su alumnado. (...) espero poder lograrlo con éxito a finales de curso para conseguir ser un hablante ideal y una buena referente para mis futuros alumnos.

La CB 2 contradice las directrices del Marco Común Europeo de Referencia (2002), porque la finalidad de la educación en lenguas ya no es el dominio de una o más lenguas, consideradas de forma aislada, con el hablante nativo ideal como modelo. El objetivo es el desarrollo del repertorio lingüístico.

CP 2.1: Para la ampliación del repertorio lingüístico es necesario aprender a navegar dentro de la diversidad lingüística.

Así pues, la ampliación de mi repertorio lingüistico se llevaba a cabo precisamente en función de los cambios en mi entorno provocados por mi trayectoria educativa, cambios de contextos, descubiertas o ampliación de horizontes, aprendiendo a navegar dentro de la diversidad lingüistica.

Esta CP 2.1 se contradice con la CB 2, porque en la creencia periférica se hace referencia al hecho de que la evolución del repertorio lingüístico está relacionada con la capacidad de saber afrontar situaciones comunicativas plurilingües, de modo que haya una transferencia de competencias en diferentes lenguas e interacción entre los conocimientos, habilidades y actitudes adquiridas en cada una de ellas.

CB 3: La mejor manera de aprender una lengua extranjera es experimentar un proceso de inmersión en la lengua meta.

La mejor manera de aprender una lengua extranjera es la de practicarla e interactuar alli donde se utiliza. 
CP 3.1: El viaje como motor básico y fundamental de aprendizaje.

Viajar es un motor básico y fundamental de aprendizaje. El conocimiento de uno mismo, la madurez, el desarrollo de habilidades sociales o los conocimientos interculturales son algunas de las habilidades que el viaje proporciona con una intensidad muy superior a la que se produce en nuestra realidad cotidiana.

CP 3.2: El aprendizaje de la L2 es una experiencia compartida: se aprende a través del uso y la interacción constante con los hablantes.

(...) el aprendizaje de 'la otra' lengua es una experiencia compartida, ya que la adopción del uso social de 'la otra' lengua depende de unos condicionantes, sobre todo de la presencia de hablantes.

En la misma línea que esta CP, las CP 1.2 y 4.2 inciden en la importancia de la presencia de hablantes y en el uso social de las lenguas para su aprendizaje.

CB 4: El desarrollo y evolución del repertorio lingüístico se realiza en función de los cambios en los diferentes contextos sociales.

Así pues, la ampliación de mi repertorio lingüistico se llevaba a cabo precisamente en función de los cambios en mi entorno provocados por mi trayectoria educativa, cambios de contextos, descubiertas o ampliación de horizontes, aprendiendo a navegar dentro de la diversidad lingüistica.

CP 4.1: Los usos de las lenguas (afectivos o funcionales) están relacionados con los ámbitos vitales y las comunidades de práctica en las que el sujeto las utiliza.

Para empezar, los idiomas que conozco son el catalán, el castellano y el inglés. El aprendizaje de estos se debe a circunstancias particulares diarias propias de la escuela, la familia o de amistades. Realmente, actualmente, con las que interactúo son la lengua castellana y la catalana. Utilizar una u otra dependerá del contexto, es decir, de las personas a quien me dirija: utilizo el castellano para comunicarme con mi familia y hablo en catalán para conversar con mis amigos o en el contexto escolar.

CP 4.2: El uso social de las lenguas permite la ampliación del repertorio lingüístico y, sobre todo, incide en el aprendizaje de segundas lenguas.

(...) el aprendizaje de 'la otra' lengua es una experiencia compartida, ya que la adopción del uso social de 'la otra' lengua depende de unos condicionantes, sobre todo de la presencia de hablantes.

CP 4.3: Es necesario que los procesos de enseñanza y aprendizaje de lenguas tengan lugar dentro del ámbito de la comunicación.

Las actividades de lengua en la escuela tendrían que ser dinámicas y pragmáticas, con la intención que el niño pueda afrontar situaciones comunicativas actuales reales. Porque 'enseñar una lengua es enseñar a utilizarla y eso sólo es posible en el ámbito de la comunicación' (Bruner, 1991).

Esta CP permite al sujeto proyectar los procesos de enseñanza y aprendizaje de lenguas en su futura docencia.

CB 5: Existen diferencias entre los usos de la L1 y la L2.

Me gustaría aclarar que para mí no lo mismo hablar en mi lengua materna que en una segunda lengua.

CP 5.1: Las habilidades, conocimientos y actitudes en L1 están más desarrolladas.

CP 5.2: Se establecen relaciones entre lengua e identidad vinculadas a las formas de conocimiento, uso y pensamiento de las lenguas.

(...) la L1 es con la que pienso, conozco mejor y con la que me comunico con mayor espontaneidad y fluidez y con menor esfuerzo y, por tanto, se trata de la lengua que prefiero utilizar y la que siento que forma parte de mi identidad; en cambio, con la L2 es todo lo contrario.

CP 5.3: La falta de hábito y de práctica en L2, tanto desde el punto de vista personal como desde el de un alumno de Primaria, dificultan el desarrollo de situaciones comunicativas.

Esta situación sería mucho más evidente en el caso de un niño de Primaria. La falta de hábito y práctica con la segunda lengua dificultarían mucho más el desarrollo de la situación comunicativa.

CB 6: Es imposible dominar una lengua, ni tan solo la materna.

Una de las muchas ideas que he interiorizado es que es imposible dominar una lengua.

CP 6.1: La lengua tiene una dimensión muy extensa y está en constante evolución.

Es prácticamente imposible dominarla, ni tan solo la lengua materna, porque se trata de una dimensión muy extensa y que está en constante evolución.

Bellaterra Journal of Teaching \& Learning Language \& Literature. 9.3 (Aug-Sept 2016)

ISSN 2013-6196 
De la narrativa visual

En la narrativa visual aparecen tres creencias base (CB), que tienen asociadas creencias periféricas (CP), que corroboran o contradicen las del relato de vida lingüística:

\section{CB 1: Las lenguas que forman parte del repertorio lingüístico están separadas en compartimientos, es decir, no hay interacción entre ellas.}

CP 1.1: No hay relación entre los conocimientos y experiencias vividas con las diferentes lenguas, de manera que difícilmente habrá transferencia de destrezas y recursos, ni se formará una competencia subyacente común.

En la narrativa visual se observa a través de las líneas divisorias que delimitan el abasto de cada lengua. Estas creencias se corroboran con la CB 2 del relato, en la que el sujeto manifiesta que en su futura profesión tendrá que ser un hablante ideal y una buena referente para sus alumnos. No obstante, se contradicen con la CB 6 de la narrativa escrita, porque en ella se afirma que una lengua es imposible de dominar por su extensa dimensión y su constante evolución.

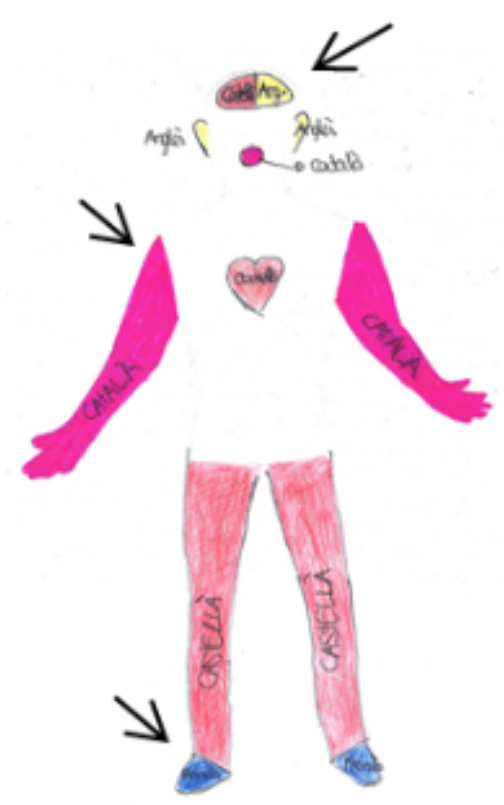

Fig. 5. Uso simbólico de las líneas para delimitar la extensión de cada lengua 
CB 2: La existencia de relaciones entre los usos de las lenguas y los ámbitos sociales en los que tienen presencia influye en las oportunidades de aprendizaje.

CP 2.1: La ampliación y evolución del repertorio lingüístico va asociada al uso social de las lenguas y a las experiencias compartidas.

Esto se manifiesta en la representación visual a través del lugar donde se sitúa cada lengua, el número de veces que aparece y el uso del color como elemento simbólico. Estas creencias están de acuerdo con la CB 1, CB 3 y CB 4 del relato, que hablan de las formas de aprendizaje de lenguas enmarcadas dentro del ámbito de la comunicación (CB 1 y 3); y de la evolución del repertorio lingüístico a través de los cambios en los contextos (CB 4).

\section{CB 3: La relación del sujeto con la $L 1$ tiene influencia en las dimensiones emocional e intelectual de la lengua.}

CP 3.1: La L1 tiene una especial incidencia en la construcción de la identidad y repercute en la relación y aprendizaje de otras lenguas.

Esto se plasma en la representación a través de la ubicación de la L1 en el corazón, la mente y en el centro. Estas creencias se relacionan con la CB 5 de la narrativa escrita, que habla de las diferencias entre los usos de la L1 y la L2.

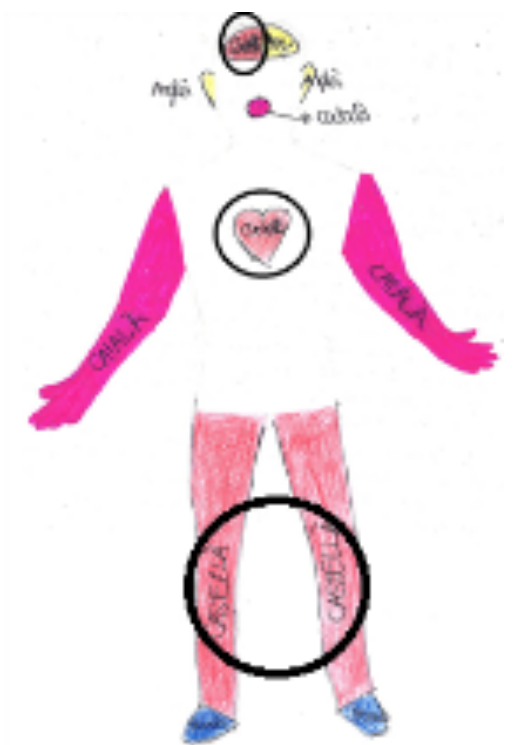

Fig. 6. Distribución de la L1 en el autorretrato lingüístico 


\section{Discusión de resultados}

Para mostrar que se han logrado los objetivos y preguntas de investigación 1 y 3 , se discuten las creencias articuladas y se ponen en relación con el marco teórico. Una de las creencias habla sobre el aprendizaje de lenguas a través de su uso, es decir, de experiencias sociales. Ésta se proyecta en las futuras prácticas docentes de la futura maestra en dos direcciones. La primera hace referencia al hecho de que 'saber una lengua' se entiende como la adquisición de competencias comunicativas; y la segunda se centra en las actividades de lengua, de las que se piensa que tienen que enmarcarse en el ámbito de la comunicación y capacitar al alumnado para afrontar situaciones comunicativas reales.

Esta primera creencia tiene implicaciones en la vida lingüística del sujeto, porque pone de manifiesto que la evolución del repertorio lingüístico está relacionada con la presencia de las lenguas en los diferentes contextos. Los resultados muestran que se establecen relaciones entre las prácticas sociales de los ámbitos escolar, familiar, lúdico y profesional, en los que se construyen y consolidan las creencias. Esto se relaciona con el hecho de que las creencias son polifónicas (Bakhtin, 1982) y resultado de procesos de interacción. Las experiencias vividas en diferentes escenarios impregnan las creencias de los individuos e influyen en la construcción del repertorio lingüístico (Bertaux, 2005). En este sentido, la futura maestra destaca la necesidad de saber de afrontar situaciones con diversidad lingüística para ampliar su repertorio lingüístico y el de sus alumnos.

En otras creencias se destaca que existen diferencias entre las prácticas y los recursos de la L1 y la L2. Una de las implicaciones que deriva de ésta pone el acento en las relaciones que se establecen entre lengua e identidad ligadas a las formas de aprendizaje, pensamiento y uso de las lenguas. Así se enfatiza la influencia de su uso en la comunidad inmediata en la formación y proyección de la identidad en la esfera social (Ricoeur, 1995).

La exploración de las relaciones entre las creencias del relato y la narrativa visual ha mostrado que la asunción de éstas no es sólida, sino que puede evolucionar gracias a procesos de reflexión (Palou y Fons, 2011). El análisis del sistema de creencias permite identificar 
contradicciones entre creencias movilizadas, las cuales conducen a su desestabilización. Por un lado, se cree que la maestra tiene que ser un hablante ideal, que se pueden dominar las lenguas del repertorio lingüístico y que éstas están separadas en compartimientos, es decir, que no hay interacción entre ellas. Esto presupone la existencia de un hablante nativo ideal que tiene como objetivo lograr el dominio de una lengua.

Por otro lado, la maestra en formación inicial piensa que es imposible dominar una lengua, por su extensa dimensión y su evolución constante. Por tanto, creencias que eran resistentes al cambio, muy arraigadas al modelo tradicional de enseñanza y aprendizaje, acontecen dinámicas, de modo que la futura maestra está en disposición de apropiarse de las teorías didácticas de referencia. El sujeto reinterpreta sus experiencias y crea nuevos marcos de interpretación (Bolívar, Domingo y Fernández, 2001), que le permiten entender que la educación en lenguas debe tener como objetivo el desarrollo del repertorio lingüístico (Marco Común Europeo de Referencia para las Lenguas, 2002).

En este proceso de interpretación de las creencias se observa la orientación de las narrativas en forma de prácticas en situación (Woods, 2006), a través de las cuales se contribuye a reproducir o transformar la educación en lenguas. Este proceso de re-negociación de las creencias es necesario, porque éstas son un marco de referencia durante la actividad pedagógica (Alanen, 2006). También se ha demostrado que las creencias arraigadas al pasado como aprendiz de lenguas tienen una mayor incidencia que la formación universitaria, aunque ésta se utilice para confirmarlas (Gabillon, 2012).

A continuación, se responde a la segunda pregunta y objetivo sobre el recurso metodológico. Las narrativas multimodales han sido una herramienta eficaz para movilizar, reflexionar y tomar conciencia de las creencias, lo que ha permitido observar si son resistentes o susceptibles al cambio. En los procesos educativos, las creencias son utilizadas como herramientas de mediación que hacen posible la atribución de significado. Por esto, se puede afirmar que las narrativas multimodales han permitido la organización y reconstrucción de las experiencias de la aprendiz de maestro, que están relacionadas con la esfera social en la que se produce la mediación (Kalaja, Dufva y Alanen, 2013). 
Las nuevas vías de investigación que se abren a partir de este trabajo son dos. La primera hace referencia a la exploración de otras formas de multimodalidad, con el propósito de observar si son tan eficaces como las utilizadas. La segunda es el estudio de las trayectorias de aprendizaje de lenguas de futuros maestros, poniendo el acento en la construcción del repertorio lingüístico en entornos plurilingües de enseñanza y aprendizaje.

Los límites de esta investigación son dos. El primero se centra en el grado de profundidad del conocimiento del sujeto, teniendo en cuenta que, a parte del relato y la narrativa visual, hubiera aumentado la validez del estudio la realización de entrevistas. Así, se habrían triangulado más datos y se habría hecho un seguimiento exhaustivo de la maestra en formación inicial. Esto hubiera permitido hacer una construcción progresiva de los componentes colectivos de la situación estudiada, de modo que se hubieran identificado resonancias, es decir, se hubiera descubierto lo general entre las formas particulares.

El segundo límite hace referencia al hecho de no obviar la influencia de la investigadora, en tanto que toda narrativa tiene en cuenta su destinatario. Esta figura influye en la interpretación particular que hace la futura maestra de los hechos que ha vivido en relación con la construcción de su repertorio lingüístico. Las experiencias que narra siempre han pasado por un filtro (Bertaux, 2005). En definitiva, los resultados muestran que la formación reflexiva e introspectiva permite utilizar la narrativa de la experiencia como herramienta para el desarrollo profesional de los aprendices de maestro.

\section{Conclusiones}

En esta investigación se han estudiado las narrativas multimodales de un sujeto, en las que ha reconstruido experiencias de relación con las lenguas, que le han permitido tomar conciencia y desarrollar el repertorio lingüístico mediante la interacción con entornos de socialización de los ámbitos familiar, social, escolar y profesional. El proceso de metacognición que ha realizado ha posibilitado que movilizara $\mathrm{y}$, en algunos casos, desestabilizara creencias vinculadas con la construcción del repertorio lingüístico. 
Los procesos reflexivos han posibilitado atribuir sentido a las experiencias y articular las propias creencias, que son parte de la cultura de enseñanza y aprendizaje de lenguas. Las creencias están vinculadas a la práctica docente porque influyen en los momentos de toma de decisiones, diseño de actividades, gestión de aula e interpretación de los acontecimientos educativos. Éstas tienen un papel destacado cuando se utilizan como herramientas de mediación, por lo que es necesaria la construcción de nuevos marcos interpretativos que permitan nuevas lecturas sobre la educación en lenguas.

Des de la perspectiva de la formación inicial de maestros, la práctica reflexiva e introspectiva permite (re)pensar las experiencias y confrontar las propias creencias durante los procesos de apropiación de las teorías didácticas de referencia. Esta práctica promueve la discusión de teorías a partir del análisis de las propias experiencias. Los futuros maestros cuestionan los modelos de socialización docentes tradicionales vividos durante su infancia, de manera que superan ciertas reticencias que pueden generar, en un primer estadio, los modelos didácticos actuales de referencia. Durante la formación universitaria se tienen que ofrecer espacios para aprender a reflexionar, de modo que se tenga un cierto control sobre lo que se hace y piensa. En este estudio se ha ofrecido la oportunidad a futuros maestros de encontrar el equilibrio que proporciona el hecho de decidir conscientemente qué tienen sentido y qué no en el propio trayecto vital, de formación y profesional.

\section{Referencias}

Alanen, R. (2006). A sociocultural approach to young language learner's beliefs about language learning. Dentro P. Kalaja y A. Ma. F. Barcelos (Eds.), Beliefs about SLA: new research approaches (pp. 55-86). Nueva York, NY: Springer.

Bakhtin, M. M. (1982). Estética de la creación verbal. México, DF: Siglo veintiuno.

Bertaux, D. (2005). Los relatos de vida. Perspectiva etnosociológica. Barcelona: Edicions Bellaterra.

Bolívar, A., Domingo, J., y Fernández, M. (2001). La investigación biográfico-narrativa en educación. Madrid: La Muralla.

Borg, S. (2003). Teacher cognition in Language teaching: a review of research on what Language teachers think, know, believe and do. Language Teaching, 36 (2), 81-109. https://doi.org/10.1017/s0261444803001903 
Consejo de Europa. (2002). Marco Común Europeo de Referencia para las Lenguas: aprender, enseñar y evaluar. Recuperado desde

http://cvc.cervantes.es/ensenanza/biblioteca_ele/marco/cvc_mer.pdf

De Fina, A., y Georgakopoulou, A. (2008). Analysing narratives as practices. Qualitative Research, 8 (3), 379-387. https://doi.org/10.1177/1468794106093634

Denzin, N. K. (2001). The reflexive interview and a performative social science. Qualitative Research, 1 (1), 23-46. https://doi.org/10.1177/146879410100100102

Gabillon, Z. (2012). Revisiting foreign language teacher beliefs. Frontiers of Language and Teaching, 3, 190-203.

Kalaja, P., Dufva, H., y Alanen, R. (2013). Experimenting with visual narratives. Dentro G. Barkhuizen (Ed.), Narratives in applied linguistics (pp. 105-131). Cambridge: Cambridge University Press.

Martucelli, D. (2013). Qu'est-ce qu'une biographie extrospective? Dentro C. Niewiadomski y C. Delory-Mamberger (Dir.), La mise en récit de soi (pp. 115-127). Lille: Presses Universitaires du Septentrion.

Melo-Pfeifer, S., y Schmidt, A. (2012). Linking 'heritage language' education and plurilingual repertories development: evidences from drawings of Portuguese pupils in Germany. L1Educational Studies in Language and Literature, 12, 1-30. https://doi.org/10.17239/11esll2012.02.11

Palou, J., y Fons, M. (2011). Historias de vida y reflexividad en los procesos de formación para la enseñanza de lenguas en entornos plurilingües. Dentro A. Lopes, F. Hernández, J. M. Sancho y J. I. Rivas (Eds.), Histórias de vida em Educação: a construção do conhecimento a partir de histórias de vida. Barcelona: Universitat de Barcelona. Recuperado desde http://hdl.handle.net/2445/47252

Pavlenko, A. (2007). Autobiographic narratives as data in applied linguistics. Applied Linguistics, 28 (2), 163-188. https://doi.org/10.1093/applin/amm008

Perregaux, C. (2011). Draw me a language! Understanding the imaginary of young children. Child Health and Education, 3 (1), 16-30.

Prasad, G. (2014). Portraits of plurilingualism in a French international school in Toronto: Exploring the role of visual methods to access students' representations of their linguistically diverse identities. The Canadian Journal of Applied Linguistics, 17 (1), 5177.

Ricoeur, P. (1995). Tiempo y narración. III: El tiempo narrado. México, DF: Siglo XXI.

Toolan, M. (2001). Narrative: A critical linguistic introduction. Londres: Routledge.

Woods, D. (2006). The social construction of beliefs in the language classroom. Dentro P. Kalaja y A. Ma. F. Barcelos (Eds.), Beliefs about SLA: new research approaches (pp. 201-230). Nueva York, NY: Springer.

Información de la autora: Míriam Cabré Rocafort es graduada en Educación Primaria (Universitat de Barcelona) y tiene un máster de Investigación en Didáctica de la Lengua y la Literatura (Premio Extraordinario de Grado y Máster). Es becaria predoctoral del Departamento de Didáctica de la Lengua y la Literatura; docente de los grados en Educación Infantil y Primaria; y miembro del grupo de investigación Plurilingüismos Escolares y Aprendizaje de Lenguas (PLURAL) de la Universitat de Barcelona.

E-mail: mcabre@ub.edu

Bellaterra Journal of Teaching \& Learning Language \& Literature. 9.3 (Aug-Sept 2016) ISSN 2013-6196 
Para citar este artículo:

Cabré Rocafort, M. (2016). Estudio de las creencias de una futura maestra sobre la construcción del repertorio lingüístico a través de narrativas multimodales. Bellaterra Journal of Teaching \& Learning Language \& Literature, 9(3): 50-68. DOI: http://dx.doi.org/10.5565/rev/jt13.652

(c) (1)

${ }_{\mathrm{BY}}$

Bellaterra Journal of Teaching \& Learning Language \& Literature. 9.3 (Aug-Sept 2016) 